\title{
ECG Signal Acquisition with on Chip Compression Algorithm
}

\author{
Rahul Kala \\ Centre for Development of Advanced \\ Computing, Mohali, Punjab, India
}

\author{
Mandeep Singh \\ Centre for Development of Advanced \\ Computing, Mohali, Punjab, India
}

\begin{abstract}
In this paper ECG signal acquisition and compression of the ECG signal is presented. ECG is an electronic method of recording the heart beat of the patient. The characteristic of heart beat is determined by PQRST curve. The detection of QRS complexes in an ECG signal provides information about the heart rate. This paper includes all the details about how to get the proper ECG signal and how it can be compressed. The paper presents an Electrocardiographic (ECG) signal front-end amplifier or pre-amplification of ECG for further processing. The signal conditioning contains amplification and filtering. Compression is required for the fast transmission of ECG signal over wireless network and to reduce the amount of data storage in long-term ECG recording. A new algorithm named Partition Algorithm is proposed for ECG signal compression and reconstruction which involves the partitioning of input signal into eight digital threshold levels which are further reconstructed. Also, it is proved that eight threshold levels are sufficient for providing the necessary ECG information in digital format.
\end{abstract}

\section{Keywords}

Front end amplifier, partition technique

\section{INTRODUCTION}

The electrocardiogram (ECG) is a recording of body surface potentials generated by the electrical activity of the heart. As recording and interpretation of the ECG has a very long history. It is an important aspect of the clinical evaluation of an individual's cardiac status and overall health. The ECG characteristics are described by the PQRST curve. The shape and size of the P-QRS-T wave and the time intervals between various peaks contains useful information about the nature of disease afflicting the heart. The $\mathrm{P}, \mathrm{QRS}$ and $\mathrm{T}$ waves reflect the rhythmic electrical depolarization and re-polarization of the myocardium associated with the contraction of Atria and Ventricles.

- The horizontal segment of the waveform preceding and $\mathrm{P}$ wave is designated as baseline for isopotential line.

- $\mathrm{P}$ wave represent the depolarization of Atria musculature.

- The QRS complex is combined result of repolarization of Atria and depolarization of Ventricles which occurs almost simultaneously.

- The $\mathrm{T}$ wave is a wave of Ventricles re-polarization.

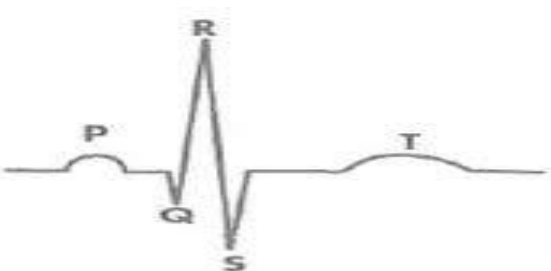

Figure1. PQRST curve

The need for compressing biomedical signals is important due to the tremendous amount of data that need to be stored efficiently at low cost [1]. The desired objective is to provide a high-quality reconstruction of electrocardiogram (ECG) signals at low bit rates and acceptable distortion levels. Mainly ECG data compression has two goals: - (1) to reduce the number of bits per sample and (2) to preserve the original signal quality [1]. The first step in the design of an ECG system involves understanding the nature of the signal that needs to be acquired. ECG signal consists of low amplitude voltages in the presence of high offsets and noise. $\mathrm{Ag} / \mathrm{AgCl}$ (Silver-Silver chloride) is the common electrode used in ECG systems and has a maximum offset voltage of $\pm 300 \mathrm{mV}$. The actual desired signal is $\pm 0.5 \mathrm{mV}$ superimposed on the electrode offset. So the system also picks up the $50 / 60 \mathrm{~Hz}$ noise from the power lines which forms the common mode signal. As amplitude of the power line noise could be very high and needs to be filtered.

\section{SIGNAL CONDITIONING OF ECG SIGNAL}

Analog Front-End processing forms a significant part of the ECG system since it needs to make a distinction between noise and which is of small amplitude having desired signal. The front-end processing circuitry consists of an instrumentation amplifier which reduces the common mode signal. The instrumentation amplifiers ought to have high input impedance since the skin resistance could be incredibly large. Operational amplifiers are required for signal conditioning of ECG device. The signal chain in support of the ECG signal conditioning consists of instrumentation amplifiers, filters implemented through op-amps or through $\mathrm{R}$ (resistor) and $\mathrm{C}$ (capacitor) component. The genuine ECG signal value is $0.2 \mathrm{mV}$ or $0.5 \mathrm{mV}$ with frequency of $0.5 \mathrm{~Hz}$ to $100 \mathrm{~Hz}$ and we require proper amplification of the signal. But during amplification, the noise is also 
amplified by the amplifier and we have to reduce the noise from the ECG signal. The main sources of noise in ECG are

- Baseline wander (low frequency noise)

- Power line interference $(50 \mathrm{~Hz}$ or $60 \mathrm{~Hz}$ noise from power lines)

- Muscle noise (This noise is very hard to remove as it is in the same section as the actual signal that is usually corrected in software.)

- Other disturbance (i.e., radio frequency noise from other equipment)

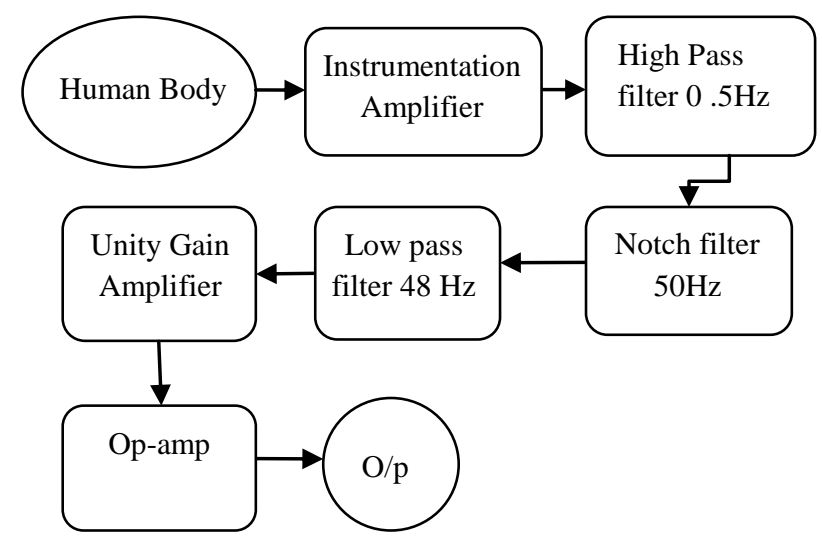

Figure2.Block schematic of a pre-amplification circuit Figure2 shows the necessary ECG front end analog processing. The Instrumentation amplifier provides difference between the ECG leads (it can be RA, LA and RL) and it attenuates the noise also. The output of the INA is quite low and we need amplification of the signal, but the amplification of the signal further amplifies the noise also, so we need filtering of the signal. Instrumentation amplifier used here is AD620 or differential op-amp INA 106 .The filtering of the signal reduce noise, therefore plan an high pass filter (with opamp or RC filter ) with cut-off frequency of $0.5 \mathrm{~Hz}$ and it may need some more stages of the high pass filter (as per the required output ). The main source of noise is power line noise. The Notch filter is used for eliminating the power line noise $(50 \mathrm{~Hz}$ or $60 \mathrm{~Hz})$. Basically Notch filter gives the zero output at the particular frequency, it basically a band pass filter $(49 \mathrm{~Hz}$ to $51 \mathrm{~Hz})$. It contains H.P.F and L.P.F called twin-T network. Designing a notch filter of $50 \mathrm{~Hz}$ frequency results in exclusion of power line noise and it also rectifies the signal. After using H.P.F and notch filter, the low frequency noise is removed by low pass filter, therefore use a low pass filter with cutoff frequency of $48 \mathrm{~Hz}$ and it may also need some more stages of the low pass filter. For providing the isolation between the circuits, an op-amp can be used as a buffer and it also advantage in removal of background noise of the signal, basically it is a unity gain amplifier. Output possibly will be in fewer amplitude, but further strengthening of the signal results into exact preferred ECG signal. The very last amplification is done by the operational amplifier which increase the strength of signal, to amplify ECG signal the gain of the associated operational amplifier must be very high.

The amplification is ended by CA-3140 BiMOS operational amplifier with MOSFET input / bipolar output is a high performance amplifier. CA3140 are integrated circuit operational amplifiers that merge the advantages of high voltage PMOS transistors with high voltage bipolar transistors on a single monolithic chip. By the appropriate use of this analog circuitry we can extract the proper ECG signal.

\section{SYSTEM APPROACH}

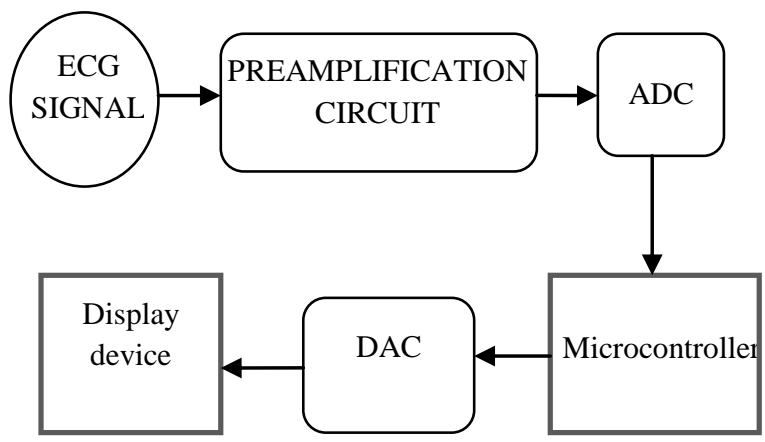

Figure3. Block schematic of a proposed system Above figure shows the block diagram of proposed system. The ECG pre-amplification circuitry is already discussed in the previous block diagram (figure no.2). The ECG signal is initially transformed into digital form by means of Analog to Digital converter (ADC) afterward it fed to the microcontroller unit. The proposed compression algorithm has been burn in microcontroller. The microcontroller ATMega32A is taken as tool for compression algorithm. The compressed ECG signal from the microcontroller is further converted into analog signal by means of digital to analog converter (DAC). The DAC 0808 is used to convert the digital signal to analog signal. Cathode ray oscilloscope (CRO) can be used as display tool.

\section{ECG DATA COMPRESSION METHODOLOGY}

After the extraction of the appropriate ECG signal, it is transformed into digital form by means of inbuilt ADC in microcontroller (ATMega32A microcontroller has inbuilt 10 bit ADC). The output of ADC is an 8 bit data it is converted into 3 bit, fundamentally it encoded 8 bit data into 3 bit. Compression is done by reducing the number of bits of the original signal. Encoding involves the use of a code to change of the original data into a form that can be used by external process. Encoding of the data shows the compression of the original ECG signal. After compressing the signal it is necessary to reconstruct the original signal. Decoding process has been used for decompressing the compressed data.

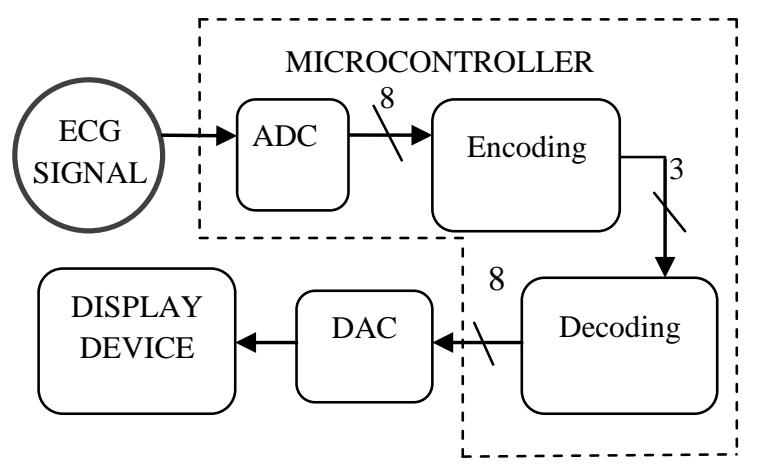


Figure4. Block schematic of a proposed ECG compression algorithm

Decoding involves the conversion of an encoded format back into the original sequence of data. In the decoding section the compressed 3 bit data is decoded into 8 bit data and this 8 bit data is further fed toward Digital analog converter (DAC). The proposed algorithm includes the process of encoding and decoding of the data. The entire process of analog to digital conversion, encoding and decoding is done in microcontroller as shown in figure4. The last section includes display unit, display device can used to see the required signal and the display equipment can be $\mathrm{CRO}$.

\subsection{Compression Algorithm}

The Proposed Algorithm for ECG Signal compression involves partitioning the input signal into some threshold voltage levels hence it is named Partition Algorithm. The Partition Algorithm involves two sections:
I. Data compression (Encoding)
II. Data reconstruction (Decoding)

\section{i. Data compression (Encoding)}

Data compression involves simply an encoding stage as shown in figure4. The 8 bit digital output from ADC (Analog to Digital Converter) can range from 00 to FF (in Hexadecimal) i.e. 0 to 255 (in decimal). This 8-bit data from the ADC is encoded into 3 bit data in encoding block. Fundamentally, it reduces the resolution of the signal by converting the 8 bit into 3 bit i.e. total number of values reduces from $2^{8}=256$ to $2^{3}=8$. Since ECG signal involves five peaks PQRST, thus eight digital values are sufficient to represent these peak values. The Digital ECG signal obtained from ADC is divided into 8 voltage threshold levels as shown in table 1 .

Table1. Different threshold levels

\begin{tabular}{|c|c|c|}
\hline $\begin{array}{c}\text { 8-bit ADC } \\
\text { output } \\
\text { (decimal) }\end{array}$ & $\begin{array}{c}\text { Compressed } \\
\text { 3-bit output } \\
\text { (Binary) }\end{array}$ & $\begin{array}{c}\text { 8-bit output to } \\
\text { DAC (decimal) }\end{array}$ \\
\hline $0-31$ & 000 & 0 \\
\hline $32-63$ & 001 & 32 \\
\hline $64-95$ & 010 & 64 \\
\hline $96-127$ & 011 & 96 \\
\hline $128-159$ & 100 & 128 \\
\hline $160-191$ & 101 & 160 \\
\hline $192-223$ & 110 & 192 \\
\hline $224-255$ & 111 & 255 \\
\hline
\end{tabular}

These levels are separated as per the output voltage range of the pre-amplification circuit. The reference voltage (Vref) of the ADC should be carefully decided. The eight voltage levels include all the peaks of the ECG PQRST curves. The 8-bit digital output of ADC are sent serially by UART and seen on PC. The digital form of the signal has some pattern of values, for example 000118063244 024106 . The maximum value of the signal represents the $\mathrm{R}$ peak of the ECG waveform (i.e. 244), 118 represents $P$ wave, 063 show $Q$ wave, 106 represents $T$ wave etc. If the value lies between different ranges then it can be taken as corresponding value of the level, suppose value
244 lies between the range of " $224-255$ " then it is taken as " 111 " and if value is " 067 " is came then it will lie between the range of "064-095" then it will taken as "011" as shown in table 1 .

\section{ii. Data Reconstruction (Decoding)}

Data reconstruction involves simply a decoding stage as shown in figure4. The 3-bit digital output from encoding stage can range from 000 to 111 (in binary) i.e. 0 to 7 (in decimal). If this 3-bit digital data is seen on CRO then we cannot easily identify the PQRST peaks. However, with a closer look and proper knowledge of ECG signal one can identify this 3-bit Signal also. As R peak has Maximum value (111) and $S$ peak has minimum value (000), therefore these are easily identified. However, we require the peaks to be clearly visible, so the 3-bit output of encoding stage is converted into 8-bit Digital format to increase the difference between various peaks and hence PQRST peaks are easily identified. Since the input to Decoding stage can range from 000 to 111 i.e. only 8 voltage values, so the original signal of 255 values is almost impossible to recover from here.

Thus, an efficient approach is to provide 8-bit eight voltage values corresponding to 3-bit eight voltage values, so that each peak clearly distinguishes from each other. This is done by providing the R-peak with Maximum value ( $F F$ in hex), $S$ peak with Minimum value (00 in hex) and the values for remaining three curves can be assigned three of the remaining six levels. The 8-bit value assigned corresponding to 3-bit input is shown in table 1 . As R and S peak is detected by the $00 \mathrm{H}$ and FFH value respectively, thus the peak exactly right to $S$ will be $T$ peak, low value peak exactly left to $R$ will be $Q$ peak and the high value peak exactly left to $Q$ peak will be $\mathrm{P}$ peak. In this way, PQRST complex is easily obtained on CRO providing the necessary information. The Partition Algorithm shows the eight digital values that are sufficient for displaying the ECG signal while at the same time providing the necessary information of patient.

\section{RESULT}

The results of the proposed algorithm can be described by its compression ratio. Compression ratio (CR) can be explain as, or calculated as

$$
\mathrm{CR}=\mathrm{D}_{\mathrm{O}} / \mathrm{D}_{\mathrm{C}}
$$

Where $D_{O}$ is the total number of bits in the original signal and $\mathrm{D}_{\mathrm{C}}$ is the total number of bits in the compressed signal [1]. 


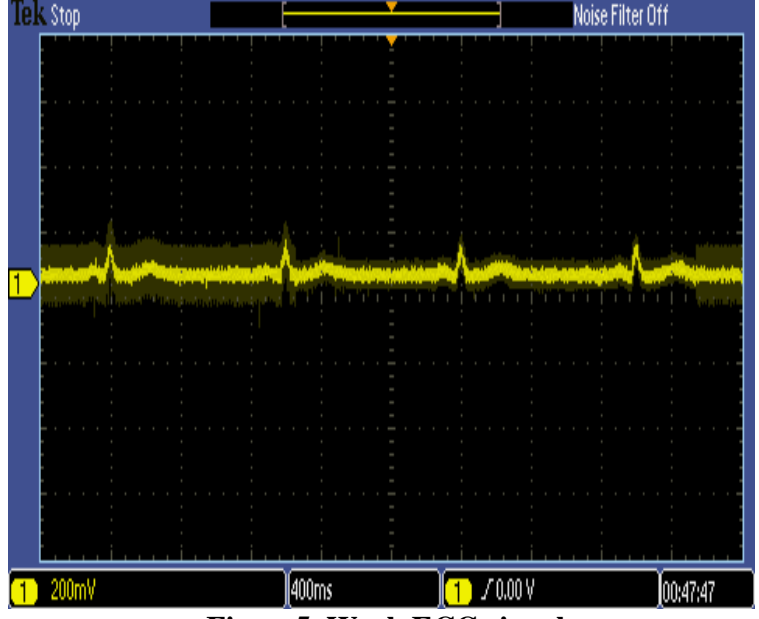

Figure5. Weak ECG signal Table2. Performance Comparison

\begin{tabular}{|c|c|}
\hline Algorithm & Compression ratio (CR) \\
\hline Proposed & 32 \\
\hline m-AZTEC & 5.6 \\
\hline Hilton [7] & 8 \\
\hline Djohan [9] & 8 \\
\hline SPIHT [10] & 8 \\
\hline VQ-Based m-AZTEC & 11.2 \\
\hline
\end{tabular}

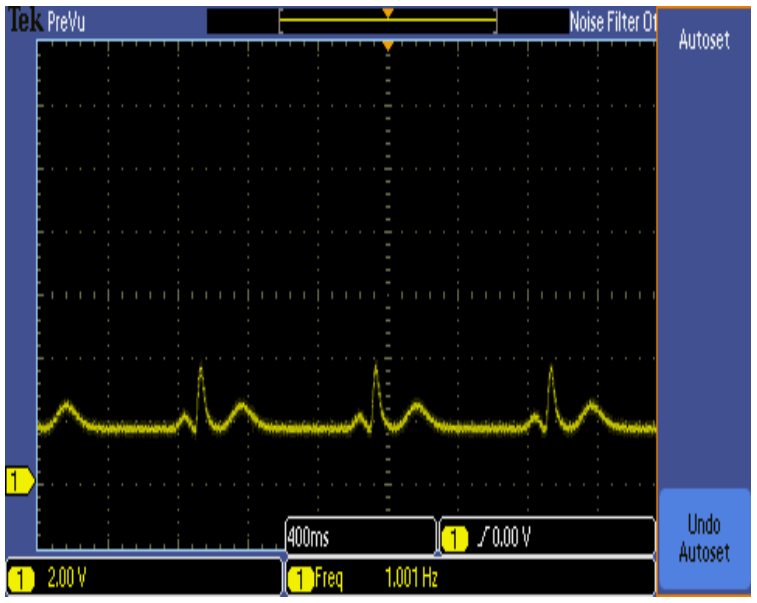

Figure6. Actual ECG signal output

\section{CONCLUSION}

An effective ECG compression algorithm is proposed in this paper. By using this proposed algorithm the obtained compression ratio is much improved than other methods. The clinical information is preserved after compression and this makes the algorithm safe to be used to compress the ECG signals. The proposed technique can achieve good compression ratio with low distortion. The simplicity makes the algorithm unique and attractive approach for mobile ECG data monitoring system.

\section{REFRENCES}

[1] Mukhopadhyay, S.K.; Mitra, M.; Mitra, S.; , "An ECG data compression method via R-Peak detection and ASCII Character Encoding," Computer, Communication and Electrical Technology (ICCCET), 2011 International Conference on, pp.136-141, 18-19 March 2011

[2] Horspool, R.N.; Windels, W.J., "An LZ approach to ECG compression," Computer-Based Medical Systems, 1994., Proceedings 1994 IEEE Seventh Symposium on, pp.71,76, 10-12 Jun 1994

[3] Arnavut, Z.; Plaku, E., "Lossless compression of ECG signals," [Engineering in Medicine and Biology, 1999. 21st Annual Conference and the 1999 Annual Fall Meetring of the Biomedical Engineering Society] BMES/EMBS Conference, 1999. Proceedings of the First Joint, vol.1, pp.274

[4] Wang Wei; Zhang Sidong, "An adaptive real-time ECG data compression algorithm," Signal Processing, 1996., 3rd International Conference on ,vol.2, pp.1719,1722

[5] Jun Meng; Ke Xu; Jun Zheng; Wenjun Yan, "Heart condition analysis from ECG signals based on nonlinear theory," Intelligent Control and Automation (WCICA), 2010 8th World Congress on , pp.1623,1628

[6] Piskorowski, J., "Powerline interference removal from ECG signal using notch filter with non-zero initial conditions," Medical Measurements and Applications Proceedings (MeMeA), 2012 IEEE International Symposium on, pp.1,3,18-19 May 2012

[7] M.L. Hilton, "Wavelet and wavelet packet compression of electrocardiograms", IEEE Trans Biomed Eng 1997;44(5):394-402.

[8] Qunyi Zhou;, "Study on ECG Data Lossless Compression Algorithm Based on K-means Cluster," Future Computer and Communication, 2009. FCC '09. International Conference on, pp.91-93, 6-7 June 2009.

[9] A. Djohan, Nguyen TQ, Tompkins WJ,"ECG compression using discrete symmetric wavelet transform”,. In: IEEE International Conference on Engineering in Medicine and Biology Society, vol. 1. 1995. p. 167-8.

[10] Z.Lu, D.Y. Kim, W.A. Pearlman., "Wavelet compression of ECG signals by the set partitioning in hierarchical trees (SPIHT) algorithm." IEEE Trans Biomed Eng 2000;47(7):849-56

[11] Sangjoon Lee; Jungkuk Kim; Jong-Ho Lee, "A Real-Time ECG Data Compression and Transmission Algorithm for an e-Health Device," Biomedical Engineering, IEEE Transactions on , vol.58, no.9, pp.2448,2455,Sept.2011

[12] Xiaoyan Qiao; Yuan Zhao; Honglei Wu, "Optional Quality Control of ECG Data Compression Algorithm," Bioinformatics and Biomedical Engineering, (iCBBE) 2011 5th International Conference on, vol., no., pp.1,4, 10-12 May 2011 
[13]ZhiLin Zhang; Tzyy-Ping Jung; Makeig, S.; Rao, B.D., "Compressed Sensing for Energy-Efficient Wireless Telemonitoring of Noninvasive Fetal ECG Via Block Sparse Bayesian Learning," Biomedical Engineering, IEEE Transactions on, vol.60, no.2, pp.300,309,Feb.2013

[14] Saberkari, H.; Shamsi, M., "Comparison of different algorithms for ECG signal compression based on transfer coding," Industrial Electronics and
Applications (ISIEA), 2012 IEEE Symposium on , vol., no., pp.341,345, 23-26 Sept. 2012

[15] Ebrahimzadeh, A.; Azarbad, M., "ECG compression using wavelet transform and three-level quantization," Digital Content, Multimedia Technology and its Applications (IDC), 2010 6th International Conference on, vol., no., pp.250,254, 16-18 Aug. 2010 